\title{
The Supersymmetric Dispersionless Systems
}

\section{Ziemowit Popowicz*}

Wroctaw University, Institute of Theoretical Physics,

Wroctaw, pl. M. Borna 9, Poland,

E-mail: 'ziemek@ift.uni.wroc.pli

Abstract: We consider in the first part, the $N=1$ supersymmetric extension of the polytropic gas dynamics. We give both the Lagrangian as well as the Hamiltonian description and construct the infinite set of "Eulerian" conserved charges associated with this system. In the second part, we present a systematic procedure for obtaining the dispersionless limit of a class of $N=1$ supersymmetric systems starting from the Lax description of their dispersive counterparts. We demonstrate this method by working out explicitly the examples of the dispersionless supersymmetric two boson hierarchy and the dispersionless supersymmetric Boussinesq hierarchy.

\section{Introduction}

Hydrodynamical systems are dispersionless systems which have been studied from a variety

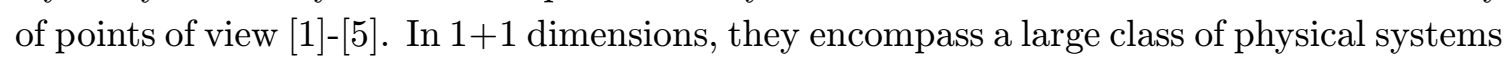

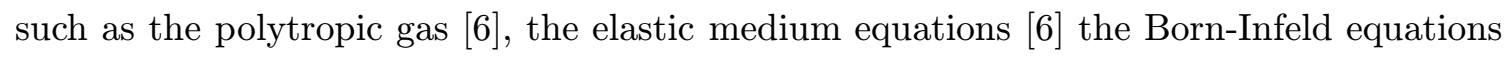
[i], ', i i i all of which are integrable. Some of these dispersionless systems [9] can be related to string theory, membrane theory and topological field [i] $\left.\overline{1}_{0}^{0}\right]$ theories.

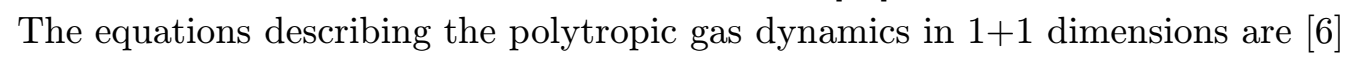

$$
\begin{array}{ll}
\frac{\partial v}{\partial t} & =(v u)_{x} \\
\frac{\partial u}{\partial t} & =\left(\frac{u^{2}}{2}+\frac{v^{\gamma-1}}{(\gamma-1)}\right)_{x}, \quad \gamma \neq 0,1
\end{array}
$$

where $u$ and $v$ are two dynamical variables belonging to the class of equations known as equations of hydrodynamical type. The system of polytropic gas equations include a large class of physical systems corresponding to different values of the exponent $\gamma$. In particular, for $\gamma=-1$, they describe the Chaplygin gas, or the Born - Infeld equation. This polytropic gas system has many similarities with the soliton equations. It possesses

\footnotetext{
${ }^{*}$ Speaker.
} 
multihamiltonian structure, has infinite number of conserved currents in involution and admits the so called Lax representation. Interestingly, the polytropic gas system can be thought as the disperssionless limit of the Kadomtsev-Petviashvilli hierarchy.

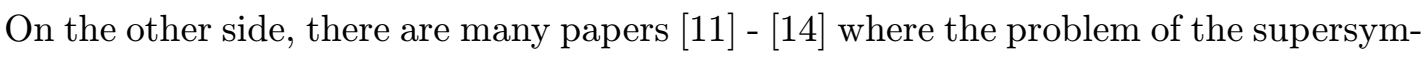
metrization of the soliton theory was considered. Therefore, the problem of constructing the supersymmetric version of the polytropic gas dynamics and the investigation of the dispersionless limit of the supersymmetric soliton theory seems to be very attractive. They

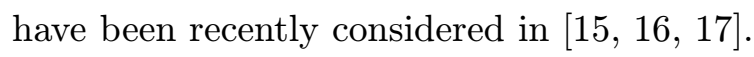

The description of the supersymmetric method used in the process of the supersymmetrization of the polytropic gas system is the main body of my first part of my talk. In the second part, we investigate the problems connected with the the supersymmetric dispersionless limit of the two boson system and Boussinesq hierarchy. These two parts are based on the common papers written together with A. Das [i] $\overline{1}$, ] and with A.Das and S.Krivonos [i 1 ind].

My talk is organized as follows. In the first section I present the basic ideas used in the soliton theory which are used in the hydrodynamical models. In the second part the idea of the supersymmetrization of the classical equations of motion is discussed. In the third section I present the supersymmetric version of the polytropic gas system considered ein [i] 1 i] $]$. In the last section the problems with the supersymmetric version of the dispersionless limit of known supersymmetric solitons equations are considered.

\section{The Polytropic gas system as a multihamiltonian system}

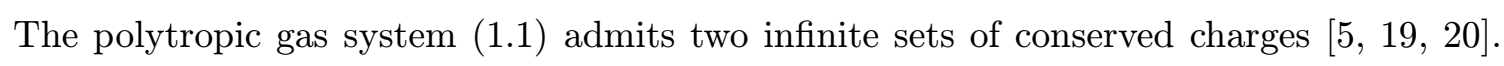
The first set, also called the "Eulerian" conserved charges [20메]

$$
H_{n}^{(1)}=\int d x h_{n}^{(1)}=\int d x \sum_{k=0}^{\left[\frac{n}{2}\right]} c(k, n) u^{n-2 k} v^{k(\gamma-1)+1}, \quad n=0,1,2, \cdots
$$

where $\left[\frac{n}{2}\right]$ stands for the integer part of the fraction and

$$
c(k, n)=\frac{1}{k !(n-2 k) !} \frac{1}{(\gamma-1)^{k}}\left(\prod_{l=0}^{k} \frac{1}{l(\gamma-1)+1}\right)
$$

Explicitly, the first four conserved charges of this infinite set has the forms

$$
\begin{aligned}
H_{0}^{(1)} & =\int d x h_{0}^{(1)}=\int d x v \\
H_{1}^{(1)} & =\int d x h_{1}^{(1)}=\int d x u v \\
H_{2}^{(1)} & =\int d x h_{2}^{(1)}=\int d x\left(\frac{1}{2 !} u^{2} v+\frac{v^{\gamma}}{\gamma(\gamma-1)}\right) \\
H_{3}^{(1)} & =\int d x h_{3}^{(1)}=\int d x\left(\frac{1}{3 !} u^{3} v+\frac{u v^{\gamma}}{\gamma(\gamma-1)}\right)
\end{aligned}
$$


The second infinite set of conserved charges, also known as the "Lagrangian" conserved charges [20 20$]$, have the following closed forms

$$
H_{n}^{(2)}=\int d x h_{n}^{(2)}=\int d x \sum_{k=0}^{\left[\frac{n}{2}\right]} c(k, n) u^{n-2 k+1} v^{k(\gamma-1)}
$$

The first three of these conserved charges have the explicit forms

$$
\begin{aligned}
& H_{0}^{(2)}=\int d x h_{0}^{(2)}=\int d x u \\
& H_{1}^{(2)}=\int d x h_{1}^{(2)}=\int d x\left(\frac{1}{2 !} u^{2}+\frac{v^{(\gamma-1)}}{(\gamma-1)(\gamma-2)}\right) \\
& H_{2}^{(2)}=\int d x h_{2}^{(2)}=\int d x\left(\frac{1}{3 !} u^{3}+\frac{u v^{(\gamma-1)}}{(\gamma-1)(\gamma-2)}\right)
\end{aligned}
$$

The system of polytropic gas equations is Hamiltonian with respect to three distinct Hamiltonian structures (the operators act on a delta function),

$$
\begin{gathered}
\mathcal{D}_{1}=\left(\begin{array}{ll}
0 & \partial \\
\partial & 0
\end{array}\right) \\
\mathcal{D}_{2}=\left(\begin{array}{cc}
\partial v^{\gamma-2}+v^{\gamma-2} \partial & \partial u+(\gamma-2) u \partial \\
(\gamma-2) \partial u+u \partial & \partial v+v \partial
\end{array}\right) \\
\mathcal{D}_{3}=\left(\begin{array}{cc}
\partial u v^{\gamma-2}+u v^{\gamma-2} \partial & \left.\partial\left[\frac{1}{2} u^{2}+\frac{1}{\gamma-1} v^{\gamma-1}\right)\right] \\
\partial\left[\frac{(\gamma-2)}{2} u^{2}+\frac{1}{\gamma-1} v^{\gamma-1}\right] & +\left[\frac{(\gamma-2)}{2} u^{2}+\frac{1}{\gamma-1} v^{\gamma-1}\right] \partial \\
+\left[\frac{1}{2} u^{2}+\frac{1}{\gamma-1} v^{\gamma-1}\right] \partial & \partial u v+u v \partial
\end{array}\right)
\end{gathered}
$$

so that we can write the polytropic gas equations as

$$
\left(\begin{array}{c}
u_{t} \\
v_{t}
\end{array}\right)=\mathcal{D}_{1}\left(\begin{array}{c}
\frac{\delta H_{2}^{(1)}}{\delta u} \\
\frac{\delta H_{2}^{(1)}}{\delta v}
\end{array}\right)=\frac{1}{\gamma} \mathcal{D}_{2}\left(\begin{array}{c}
\frac{\delta H_{1}^{(1)}}{\delta u} \\
\frac{\delta H_{1}^{(1)}}{\delta v}
\end{array}\right)=\mathcal{D}_{3}\left(\begin{array}{c}
\frac{\delta H_{0}^{(1)}}{\delta u} \\
\frac{\delta H_{0}^{(1)}}{\delta v}
\end{array}\right)
$$

There exists a simple connection between the hameiltonian operators $\mathcal{D}$ and the Poisson brackets

$$
\left\{V_{i}(x), V_{j}(y)\right\}_{n}=\mathcal{D}_{n, i, j} \delta(x, y)
$$

where $\delta$ is the Dirac delta function, $V_{i}$ are fields and $n$ enumerates different hamiltonian structures.

The previous connection create also the connection between different algebras. Notice that for $\gamma=4$ the second hamiltonian operator $\mathcal{D}_{2}\left({ }_{2}^{2} \cdot{ }^{-6}\right)$ generate the so called centerless Zamolodchikov $W_{3}$ algebra. 
The knowledge of the multihamiltonian structures is very useful because in many cases it allows to construct the recursion operator. In our case we have the the following recursion operator

$$
R=\mathcal{D}_{1}^{-1} \mathcal{D}_{2}
$$

This opertaor, as the hereditary operator [īi] densities. Namely we have

$$
\left(\begin{array}{l}
\frac{\delta H_{m+1}^{(1)}}{\delta u} \\
\frac{\delta H_{m+1}^{(1)}}{\delta v}
\end{array}\right)=\frac{1}{\gamma} \mathcal{D}_{1}^{-1} \mathcal{D}_{2}\left(\begin{array}{c}
\frac{\delta H_{m}^{(1)}}{\delta u} \\
\frac{\delta H_{m}^{(1)}}{\delta v}
\end{array}\right)
$$

The three Hamiltonian structures in Eq. (2.6.) are compatible, which can be easily seen from the fact that under the shift $u \rightarrow u+\lambda$, where $\lambda$ is an arbitrary constant, $\mathcal{D}_{2} \rightarrow \mathcal{D}_{2}+\lambda(\gamma-1) \mathcal{D}_{1}$ and $\mathcal{D}_{3} \rightarrow \mathcal{D}_{3}+\lambda \mathcal{D}_{2}+\frac{\lambda^{2}(\gamma-1)}{2} \mathcal{D}_{1}$. The compatibility of a multi-Hamiltonian structure guarantees the complete integrability of a system of dynamical equations. However, in the case of the polytropic gas equations, one can check from Eqs. $\left(\overline{2}, 1, \overline{2}, \overline{4}{ }_{1}\right)$ that the conserved densities satisfy

$$
\frac{\partial^{2} H_{n}^{(i)}}{\partial v \partial v}=v^{\gamma-3} \frac{\partial H_{n}^{(i)}}{\partial u \partial u}, \quad i=1,2 \text { and } \quad n=0,1,2, \cdots
$$

Using this, one can prove, in an elegant manner, that the conserved quantities are in involution with respect to the three Hamiltonian structures, therefore showing the complete integrability of the polytropic gas equation. Moreover this equation can be described by the Lax function [in ${ }^{1} \bar{g}_{1}^{n}$.

Let us next use the involution of the conserved quantities to derive some relations which will be useful within the context of the supersymmetric polytropic gas. Note that the involution of the charges with respect to the first Hamiltonian structure implies that

$$
\left\{H_{n}^{(1)}, H_{m}^{(1)}\right\}_{1}=\int d x\left(\frac{\delta H_{n}^{(1)}}{\delta u} \partial \frac{\delta H_{m}^{(1)}}{\delta v}+\frac{\delta H_{n}^{(1)}}{\delta v} \partial \frac{\delta H_{m}^{(1)}}{\delta u}\right)=0
$$

An explicit calculation of the Poisson bracket yields

$$
\int d x \sum_{s=0}^{\left[\frac{n}{2}\right]+\left[\frac{m}{2}\right]} \frac{u^{m+n-2 s-2} v^{s(\gamma-1)+1} u_{x}}{s(\gamma-1)+1} \sum_{l=\max \left(0, s-\left[\frac{n}{2}\right]\right)}^{\min \left(s,\left[\frac{m}{2}\right]\right)} f(s, l, n, m)
$$

where

$$
\begin{aligned}
f(s, l, n, m)= & c(s-l, n) c(l, m)[-(n-2(s-l))(n-2(s-l)-1)(l(\gamma-1)+1)(l(\gamma-1)) \\
& +(m-2 l)(m-2 l-1)((s-l)(\gamma-1)+1)((s-l)(\gamma-1))]
\end{aligned}
$$

The vanishing of the Poisson bracket, then, leads to the relation

$$
\sum_{l=\max \left(0, s-\left[\frac{n}{2}\right]\right)}^{\min \left(s,\left[\frac{m}{2}\right]\right)} f(s, l, n, m)=0
$$


Finally, let us note that it is easy to verify that the Miura transformation

$$
v=r p, \quad u=\frac{1}{(\gamma-1)}\left(r^{(\gamma-1)}+p^{(\gamma-1)}\right)
$$

maps the polytropic gas equations to the equations

$$
\begin{aligned}
\frac{d r}{d t} & =\frac{1}{(\gamma-1)}\left(r^{(\gamma-1)}+p^{(\gamma-1)}\right) r_{x}+r p^{(\gamma-2)} p_{x} \\
\frac{d p}{d t} & =\frac{1}{(\gamma-1)}\left(r^{(\gamma-1)}+p^{(\gamma-1)}\right) p_{x}+p r^{(\gamma-2)} r_{x}
\end{aligned}
$$

which can be written as Hamiltonian equations with the first structure in Eq. (5) and

$$
H=\int d x \frac{1}{\gamma(\gamma-1)}\left(p r^{\gamma-1}+r p^{\gamma-1}\right)
$$

Moreover, this Miura transformation transforms the canonical Hamiltonian operator $\mathcal{D}_{1}$ onto the second Hamiltonian operator $\mathcal{D}_{2}$.

\section{How we would like to supersymmetrize the classical systems}

In the last years, there areincreasing interest in the supersymmetric integrable systems. In particular, the supersymmetric KdV - type equations were extensively studied. However up to now we have not an unique prescription for the supersymmetrization of the classical systems. We can encounter two different methods of supersymmetrization. The first is the Lie superalgebraic approach and the second which I call as an algebraic method. Both these methods have own advantages and disadvantages.

In the first approach we replace the classical symmetry of the given system by its supersymmetrical partner. For example, starting from the supersymmetric generalizations of the Virasoro algebra and the corresponding hamiltonian structure, it was possible to construct integrable supersymmetric extensions of the classical Korteweg de Vries (KdV) equation. Lie superalgebraic approaches to the supersymmetric hierarchies were developed by Inami, Kanno and others. The classical background from which these approaches originate is the Drinfeld-Sokolov theory, concerning the relations between simple Lie algebras and KdV-type hierarchies. However, this method does not allow to obtain all possible supersymmetric extensions and in many cases it is restricted to the so called fermionic extensions of the classical systems. For example it appeared recently, that the famous KdV equation could be supersymmetrized using the so called odd Poisson brackets [2] $2 \overline{8} \overline{1}]$. Moreover, during the supersymmetrization procedure, some typical supersymmemtrical effects, not encountered in the classical case, have occurred. Interestingly, the first hamiltonian structure for the supersymmetric $N=1 \mathrm{KdV}$ equation, introduced by Manin-Radul, is the so called inverse hamiltonian while its classical counterpart is the usual one.

The second method is based on the observation that the idea of supersymmetrization consists in extending a system of $k$ bosonic equations by $k N$ fermion and $k(N-1)$ boson fields $(k=1,2, \ldots, N=1,2, .$.$) in such a way that the final theory becomes supersym-$ metrical invariant. In order to add these fields we can use the dimensional analysis and 
consider all possible supersymmetrical combinations of the given dimensional weight. Let me illustrate this method in the $\mathrm{KdV}$ equation case.

The classical KdV equation which we consider has the form

$$
\frac{\partial u}{\partial t}=-u_{x x x}+6 u u_{x}
$$

We can associate the following weights to $[u]=2,[\partial]=1,[t]=-3$, from which we conclude that the dimension of the $\mathrm{KdV}$ equation is 5 .

In the extended $N=2$ supersymmetric case we deal with the supermultiplet $\Phi$ whose Taylor expansion with respect to the two anticommuting variables $\theta_{1}$ and $\theta_{2}$ is

$$
\Phi=u_{0}+\theta_{1} \xi_{1}+\theta_{2} \xi_{2}+\theta_{2} \theta_{1} u_{1}
$$

where the fields $u_{0}, u_{1}$ are interpreted as bosonic (fermionic) fields for a superboson (superfermion) field and $\xi_{1}, \xi_{2}$ as fermions (bosons) for a superboson (superfermion) respectively. In the $\mathrm{KdV}$ case we can assume that $u_{1}=u$. If we associate the weight $\left[\xi_{i}\right]=3 / 2$ we obtain that $[\Phi]=1$ and hence the most general ansatz on the 4 dimensional combinations is

$$
\begin{aligned}
\frac{\partial \Phi}{\partial t}= & s_{1}\left(D_{1} D_{2} \Phi_{x x}\right)+s_{2} \Phi\left(D_{1} D_{2} \Phi_{x}\right)+s_{3}\left(D_{1} D_{2} \Phi\right)^{2}+s_{4} \Phi_{x}\left(D_{1} D_{2} \Phi\right)+ \\
& s_{5}\left(D_{1} D_{2} \Phi\right) \Phi^{2}+s_{6} \Phi_{x x x}+s_{7} \Phi_{x x} \Phi+s_{8}\left(\Phi_{x}\right)^{2}+s_{9} \Phi_{x} \Phi^{2}+s_{10} \Phi^{4} \\
& s_{11}\left(D_{2} \Phi_{x}\right)\left(D_{2} \Phi\right)+s_{12}\left(D_{2} \Phi_{x}\right)\left(D_{1} \Phi\right)+s_{13}\left(D_{2} \Phi\right)\left(D_{1} \Phi_{x}\right) \\
& s_{14}\left(D_{2} \Phi\right)\left(D_{1} \Phi\right) \Phi+s_{15}\left(D_{1} \Phi_{x}\right)\left(D_{1} \Phi\right)
\end{aligned}
$$

where $s_{i}$ are arbitrary constants and the superderivatives are defined as

$$
D_{1}=\partial_{\theta_{1}}+\theta_{1} \partial \quad D_{2}=\partial_{\theta_{2}}+\theta_{2} \partial
$$

with the properties

$$
D_{2} D_{1}+D_{1} D_{2}=0, \quad D_{1}^{2}=D_{2}^{2}=\partial
$$

Below we shall use the following notation: $\left(D_{i} F\right)$ denotes the outcome of the action of the superderivatives, while $D_{1} F$ denotes the action itself.

The main problem now is how to fix the constants $s_{i}$. We can do it assuming that 1.) In the bosonic sector, where all fermions and additional boson fields disappear, our equation reduces to the $\mathrm{KdV}$ equation. 2.) Our supersymmetric equation (3.3) is invariant under the rotation $D_{1} \Leftrightarrow D_{2}$. 3.) The equation (3.3) could be written down as a hamiltonian equation. 4.) Our supersymmetric equation has all or some soliton's properties, namely it is completely integrable, possesses a recursion operator and a Lax representation.

The first two assumptions fix four constants. On the other hand assuming that our equation is hamiltonian and its second hamiltonian structure is connected with the supersymmetric Virasoro algebra

$$
\frac{\partial \Phi}{\partial t}=\left(D_{1} D_{2} \partial+2 \partial \Phi+2 \Phi \partial-D_{1} \Phi D_{1}-D_{2} \Phi D_{2}\right) \frac{\delta\left(\left(D_{1} D_{2} \Phi\right) \Phi+a(\Phi)^{3}\right)}{\partial_{\Phi}}
$$


we obtain a one parameter family of equations enumerated by the constants $a$. Additional assumptions on the integrability fix constants to $a=1,-2,4$.

However in many cases we do not known the supersymmetrical version of the hamiltonian operator. We can fix this operator, also using the dimensional analysis and assuming the most general even or odd supersymmetrical hamiltonian or inverse hamiltonian structure. This is the method we use in the next section.

The disadvantage of this algebraic method consists in its complexity. The ansatz we are dealing here contains a huge number of fields and therefore we are forced to use the computer algebra in order to fix the constants [1] ${ }_{1}^{1}$. Anaothee disadvantage of this method is its restriction to dimensional fields but we do not know in fact how to apply it to zero dimensional fields.

\section{4. $N=1$ Supersymmetric polytropic gas:}

We consider here only the non-extended supersymmetrization of the polytropic gas for arbitrary $\gamma$. We have chosen to work directly at the level of the equations of motion and use our second method of supersymmetrization.

From the discussion of the last section, in particular Eq. (1, $(1)$, we note that we can assign the following dimensions

$$
[x]=-1, \quad[v]=2, \quad[u]=\gamma-1, \quad[t]=-\gamma
$$

In generalizing Eq. (1, 1יis ) to the $N=1$ supersymmetric system, we work in superspace and enlarge the number of dynamical variables to write them in terms of two fermionic superfields of the forms

$$
U(x, \theta)=\eta(x)+\theta u(x), \quad V(x, \theta)=\xi(x)+\theta v(x)
$$

where $\eta, \xi$ represent the dynamical fermionic variables, which are the superpartners of the original bosonic variables, $u, v$, respectively.

The dimensions of $[\theta]=-\frac{1}{2}$ forces the dimensions of the dynamical superfields to be

$$
[U]=\gamma-\frac{3}{2}, \quad[V]=\frac{3}{2}
$$

It is clear now that, since $\left[U_{t}\right]=2 \gamma-\frac{3}{2}$ and $\left[V_{t}\right]=\gamma+\frac{3}{2}$, in generalizing the polytropic gas equations to the superspace, we can allow for all possible local terms (involving $U,(D U), U_{x}, \cdots$ and $\left.V,(D V), V_{x}, \cdots\right)$ in the dynamical equations on the superspace, which conform to the appropriate dimensions. Furthermore, we also require that the equations be such that, in the bosonic limit, they reduce to the equations for the polytropic gas dynamics. Once we have the dynamical equations it can be determined that the system possesses an infinite set of bosonic conserved charges which similarly conforms to the appropriate dimensions and reduces to the classical charges in the bosonic limit.

This restricts the structure of the equations greatly. Interestingly, we do not obtain any equation and conserved charges if we assume the existence of the both supersymmetrical 
analog of the „, Eulerian " and „Lagrangian " charges. We did not obtain any supersymmetrical equation assuming the existence the supersymmetrical analog of the "Lagrangian" charges only.

The assumption of the existence of the supersymmetrical analogs of the "Eulerian" charges only leads us to the following equation

$$
\begin{aligned}
V_{t} & =(V(D U))_{x} \\
U_{t} & =D\left[\frac{1}{2}(D U)^{2}+\frac{1}{(\gamma-1)}(D V)^{\gamma-1}-\frac{(\gamma-2 \kappa)}{\gamma} V V_{x}(D V)^{\gamma-3}\right]
\end{aligned}
$$

where $\kappa$ is an arbitrary parameter.

The supersymmetrical analogs of the "Eulerian" conserved charges is

$$
H_{n}^{(1)}=\int d z \tilde{h}_{n}^{(1)}=\int d z \sum_{k=0}^{\left[\frac{n}{2}\right]} c(k, n) V(D V)^{k(\gamma-1)}(D U)^{n-2 k}
$$

where $d z=d x d \theta$ represents the integration over the superspace and $c(k, n)$ 's denote the constants defined in Eq. (2.2.2). These charges reduce in the bosonic limit to the "Eulerian" conserved charges in Eq. (12.1). The first ones have the following explicit forms for the first few.

$$
\begin{aligned}
H_{0}^{(1)} & =\int d z \tilde{h}_{0}^{(1)}=\int d z V \\
H_{1}^{(1)} & =\int d z \tilde{h}_{1}^{(1)}=\int d z V(D U) \\
H_{2}^{(1)} & =\int d z \tilde{h}_{2}^{(1)}=\int d z V\left[\frac{1}{2}(D U)^{2}+\frac{(D V)^{\gamma-1}}{\gamma(\gamma-1)}\right] \\
H_{3}^{(1)} & =\int d z \tilde{h}_{3}^{(1)}=\int d z V\left[\frac{1}{3 !}(D U)^{3}+\frac{1}{\gamma(\gamma-1)}(D V)^{\gamma-1}(D U)\right]
\end{aligned}
$$

These "Eulerian" charges can be easily checked to be conserved for any value of $\kappa$. In

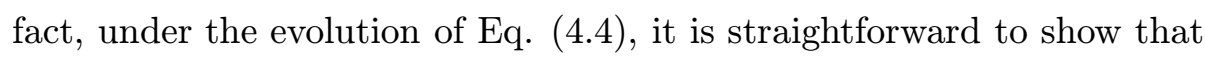

$$
\begin{aligned}
\frac{d H_{n}^{(1)}}{d t}=\int d z\left[-\sum_{k=1}^{\left[\frac{n}{2}\right]} c(k, n) \frac{k(\gamma-1)(k(\gamma-1)+1)}{n-2 k+1} V\left(D V_{x}\right)(D V)^{k(\gamma-1)}(D U)^{n-2 k+1}\right. \\
\\
\left.\quad+\sum_{k=0}^{\left[\frac{n}{2}\right]-1} c(k, n)(n-2 k) V\left(D V_{x}\right)(D V)^{(k+1)(\gamma-1)-1}(D U)^{n-2 k-1}\right] \\
=\int d z \sum_{k=1}^{\left[\frac{n}{2}\right]}\left[-\frac{k(\gamma-1)(k(\gamma-1)+1)}{n-2 k+1} c(k, n)+(n-2 k+2) c(k-1, n)\right] \\
\quad \times V\left(D V_{x}\right)(D V)^{k(\gamma-1)}(D U)^{n-2 k+1} \\
=0 \quad
\end{aligned}
$$

where we have used the fact that the quantity in the square bracket vanishes due to the structure of $c(k, n)$ (see Eq. $(\overline{2} \cdot \overline{2} \cdot \overline{2})$ ). 
Once we have the conserved quantities, we can think of them as Hamiltonians and look for a Hamiltonian description of the system. It turns out that the system of equations in Eq. (1.4.) has a Hamiltonian description only for $\kappa=1$. Namely, with the Hamiltonian structure

$$
\mathcal{D}_{1}=\left(\begin{array}{cc}
0 & D \\
D & 0
\end{array}\right)
$$

the $N=1$ supersymmetric polytropic gas equations can be written in the Hamltonian form

$$
\left(\begin{array}{c}
U_{t} \\
V_{t}
\end{array}\right)=\mathcal{D}_{1}\left(\begin{array}{c}
\frac{\delta H_{2}^{(1)}}{\delta U^{(1)}} \\
\frac{\delta H_{2}^{(1)}}{\delta V}
\end{array}\right)
$$

only if $\kappa=1$. This, therefore, selects out the particular value of the arbitrary parameter $\kappa$. However, integrability is yet to be shown, even though the presence of an infinite number of conserved quantities is suggestive.

We would choose $\kappa=1$ from now on, so that the $N=1$ supersymmetric polytropic gas equations have the form

$$
\begin{aligned}
V_{t} & =(V(D U))_{x} \\
U_{t} & =D\left[\frac{1}{2}(D U)^{2}+\frac{1}{(\gamma-1)}(D V)^{\gamma-1}-\frac{(\gamma-2)}{\gamma} V V_{x}(D V)^{\gamma-3}\right]
\end{aligned}
$$

Unlike the bosonic system, where there are three distinct Hamiltonian structures, the supersymmetric equations in (4.10i) do not seem to allow any other Hamiltonian structure (at least, we have not succeeded in finding them).

We have not managed to find an elegant proof of the involution of charges as in the bosonic case. Instead, we can show by brute force that the charges are in involution with respect to the Hamiltonian structure $\mathcal{D}_{1}$, namely, it can be shown by direct calculations that

$$
\begin{aligned}
\left\{H_{n}^{(1)}, H_{m}^{(1)}\right\}=\int d z \sum_{s=0}^{\left[\frac{n}{2}\right]+\left[\frac{m}{2}\right]} \frac{\left(V\left(D U_{x}\right)(D V)^{s(\gamma-1)}+V_{x} V U_{x}(D V)^{s(\gamma-1)-1}\right)}{s(\gamma-1)+1} \\
\quad \times(D U)^{m+n-2 s-2} \sum_{l=\max \left(0, s-\left[\frac{n}{2}\right]\right)}^{\min \left(s,\left[\frac{m}{2}\right]\right)} f(s, l, n, m) \\
=0
\end{aligned}
$$

where $f(s, l, n, m)$ is defined in Eq. $(2.15)$ and we have used the identity in Eq. (2.16i) in the final step. This shows that the infinite set of charges are in involution, thereby proving the complete integrability of the system.

As we have seen in the bosonic case, the polytropic gas equations possess two infinite sets of conserved charges, although only one set of infinite conserved charges is enough to prove integrability. It is worth inquiring about the second infinite set of conserved charges in the case of the supersymmetric polytropic gas equations. Unfortunately, we have found 
only one other charge, namely,

$$
H_{0}^{(2)}=\int d z \tilde{h}_{0}^{(2)}=\int d z U
$$

which is conserved under the evolution of the supersymmetric polytropic gas system. Of course, it reduces, in the bosonic limit, to the bosonic charges $H_{0}^{(2)}$ in Eq. (2.74).

Let us comment now on why our supersymmetric polytropic gas system has only one local Hamiltonian structure and possesses only the supersymmetric "Eulerian" series of local conserved charges (and not a second infinite set of conserved charges). First, note that the bosonic polytropic gas equation $\left(\overline{1} \overline{1}_{1}\right)$, for $\gamma=4$, can be thought of as the dispersionless limit of the Boussinesq equation. On the other hand, the second Hamiltonian structure of this equation (see Eq. (2.6i) ), corresponds to the $W_{3}$ algebra. It is well known that there is no nontrivial $N=1$ supersymmetric extension of the Boussinesq equation as well as the $W_{3}$ algebra. From the nonexistence of supersymmetric extension of $W_{3}$ algebra alone, we cannot, of course, conclude that there are no possible supersymmetrizations in the dispersionless limit. However, using computer and symbolic computations, we have checked that it is impossible to find such local structures as well as the supersymmetric analog of the Miura transformation, Eq. (2.16), in the dispersionless limit. The nonexistence of the second Hamiltonian structure, for the supersymmetric polytropic gas, also implies that there does not exist a recursion operator that is factorizable and can be written in terms of two Hamiltonian structures. This, however, does not affect the integrability of the system, as we have explicitly demonstrated that the $N=1$ supersymmetric polytropic gas system is completely integrable.

We did not find the supersymmetric analog of the Lax operator for our supersymmetric polytropic gas system. The reason will be explained in the next section.

\section{The Lax description of the dispersionless two bosons and Boussinesq hierarchy}

We describe the basic procedure of taking the dispersionless limit of bosonic systems within the framework of the Lax description itself. Let us consider a general Lax operator of the form

$$
L=\partial^{n}+\sum_{m=1}^{\infty} A_{m} \partial^{n-m}
$$

where the coefficients, $A_{m}$ 's, are functions of the dynamical variables of the system which depends on the coordinates $(x, t)$. Let us assume that the Lax equation

$$
\frac{\partial L}{\partial t_{k}}=\left[\left(L^{k}\right)_{\geq s}, L\right], \quad s=0,1,2
$$

describes the dynamical system of equations. Here ()$_{\geq s}$ represents the projection with respect to the powers of $\partial$. In going to the dispersionless limit, first of all, we replace $\partial \rightarrow p$. Then, we scale $p \rightarrow \alpha p$ and all the basic dynamical variables as $J_{i} \rightarrow(\alpha)^{i} J_{i}$ where $J_{i}$ represents the basic dynamical variables of the system with the respective dimensions 
$i$. The Lax function which describes the dispersionless system of equations is obtained as

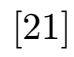

$$
\mathcal{L}=\lim _{\alpha \rightarrow \infty} \frac{1}{\alpha^{n}} L_{\alpha}
$$

where $L_{\alpha}$ denotes the scaled Lax function. The dispersionless equations are then obtained from the Lax equation

$$
\frac{\partial \mathcal{L}}{\partial t_{k}}=-\left\{\left(\mathcal{L}^{k}\right)_{\geq s}, \mathcal{L}\right\}
$$

where

$$
\{A, B\}=\frac{\partial A}{\partial x} \frac{\partial B}{\partial p}-\frac{\partial A}{\partial p} \frac{\partial B}{\partial x}
$$

represents the Poisson bracket on the classical phase space.

Let us illustrate this procedure with a few examples. The reduction of the KdV equation to its dispersionless limit is well known and, therefore, we will not repeat it here. As a first example lest us consider the following Lax operator

$$
L:=\partial^{3}+v \partial+u
$$

and its Lax pair representation

$$
\frac{\partial \mathcal{L}}{\partial t}=\left[\left(\mathcal{L}^{\frac{4}{3}}\right)_{\geq 0}, \mathcal{L}\right]
$$

The last equation generates the following equations

$$
\begin{aligned}
\frac{\partial u}{\partial t}= & \left(-3 u_{x x x}-12 u_{x} u+2 v_{5 x}+6 v_{x x x} v+12 v_{x x} v_{x}\right. \\
& \left.-6 v_{x} u_{x}+4 v_{x} v^{2}-6 v u_{x x}\right) / 9 \\
\frac{\partial v}{\partial t}= & \left(-2 u_{x x x}+v_{4 x}+2 v_{x x} v+2 v_{x}^{2}-4 v_{x} u-4 v u_{x}\right) / 3
\end{aligned}
$$

In the dispersionless limit these equations reduce to the polytropic gas system dynamics while the Lax operator reduces to

$$
L=p^{3}+v p+u
$$

Let us look now at the two boson hierarchy [i2 $2 \overline{2}]$ described by the Lax operator

$$
L=\partial-J+\partial^{-1} T
$$

with the nonstandard Lax equation given by

$$
\frac{\partial L}{\partial t_{k}}=-\left[\left(L^{k}\right)_{\geq 1}, L\right]
$$

Here $J$ and $T$ are dynamical field variables with dimensions one and two respectively. Therefore, under the scaling discussed earlier, we have for the present case, $p \rightarrow \alpha p, J \rightarrow$ $\alpha J, T \rightarrow \alpha^{2} T$. It follows that in the dispersionless limit the Lax operator goes into

$$
\mathcal{L}=p-J+T p^{-1}
$$


and the Lax equation

$$
\frac{\partial \mathcal{L}}{\partial t_{k}}=\left\{\left(\mathcal{L}^{k}\right)_{\geq 1}, \mathcal{L}\right\}
$$

describes the dispersionless system of equations.

The two boson hierarchy can also be alternatively described in terms of the gauge

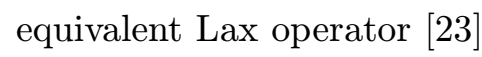

$$
L=\partial-\frac{1}{\partial+J}\left(\frac{T}{2}\right)
$$

and the standard Lax equation

$$
\frac{\partial L}{\partial t_{k}}=\left[\left(L^{k}\right)_{\geq 0}, L\right]
$$

This description of the system of equations is more convenient from the point of view of our subsequent discussions. We note that the second flow

$$
J_{t_{2}}=\left(J_{x}+T-J^{2}\right)_{x}, \quad T_{t_{2}}=-T_{x x}-2(J T)_{x}
$$

is the two boson equation which is also related to the nonlinear Schrödinger equation (NLS) [203in. The third flow, on the other hand, is obtained to be

$$
J_{t_{3}}=J_{x x x}+\left(J^{3}-3 J T-3 J J_{x}\right)_{x}, \quad T_{t_{3}}=T_{x x x}+3\left(J^{2} T-\frac{1}{2} T^{2}+J T_{x}\right)_{x}
$$

and this coincides with the bosonic sector of the $N=2$ supersymmetric KdV equation

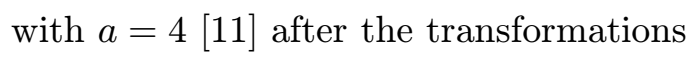

$$
J \rightarrow 2 J, \quad T \rightarrow-2\left(T+J_{x}\right), \quad x \rightarrow i x, \quad t \rightarrow i t .
$$

In the present case, it is easy to check that, in the dispersionless limit, the Lax function becomes

$$
\mathcal{L}=p-\frac{1}{p+J}\left(\frac{T}{2}\right) .
$$

The second and the third flow equations following from the Lax equation

$$
\frac{\partial \mathcal{L}}{\partial t_{k}}=-\left\{\left(\mathcal{L}^{k}\right)_{\geq 0}, \mathcal{L}\right\}
$$

are given by

$$
\begin{aligned}
& J_{t_{2}}=\left(T-J^{2}\right)_{x}, \quad T_{t_{2}}=-2(J T)_{x} \\
& J_{t_{3}}=\left(J^{3}-3 J T\right)_{x}, \quad T_{t_{3}}=3\left(J^{2} T-\frac{1}{2} T^{2}\right)_{x}
\end{aligned}
$$

These are indeed the correct dispersionless limits of the two boson hierarchy.

While the properties of such bosonic models are quite well understood, much remains to be learnt about their supersymmetric counterparts. For example, the Lax description of only a handful of supersymmetric dispersionless systems have been constructed, to this 
date, by brute force [2] $\left.\overline{2}_{-}, \overline{2} \overline{2} \overline{5}\right]$. We did not find, using brute force, the supersymmetric Lax operator for the polytropic gas system also. On the other side, there is no systematic procedure for obtaining them starting from the corresponding supersymmetric dispersive Lax descriptions. Similarly, supersymmetric dispersionless systems often have more conserved charges than their dispersive counterparts and we do not yet know how to relate the new charges to the Lax function itself.

We take a modest step here and show how one can obtain, systematically, a Lax description for a selected class of supersymmetric dispersionless systems starting from their dispersive counterparts. This ancestor $N=2$ supersymmetric systems are described in terms of the Lax operators that involve only "bosonic" operators $(\partial)$. We define in the next section the basic procedure for taking the dispersionless limit in the Lax description itself, within the context of bosonic models. We work out some known examples to illustrate the procedure and present the Lax description of some new bosonic models. We work out explicitly the example of the dispersionless supersymmetric two boson hierarchy starting from the $N=2$ supersymmetric KdV hierarchy. We extend the analysis and discuss the dispersionless supersymmetric Boussinesq.

\section{Supersymmetric KdV and two boson hierarchy}

In this section, we will generalize the ideas of the previous section to construct the Lax description for a class of dispersionless supersymmetric systems. In particular, we will work out in detail the case of the dispersionless $N=1$ supersymmetric two boson hierarchy starting from the Lax description of the $N=2$ supersymmetric KdV hierarchy with $a=4$ [26], whose bosonic sector we have studied in the last section. Let us first note that the few dispersionless supersymmetric systems [2] $2 \overline{4}$ ind whose Lax descriptions have been constructed by brute force show that, although the Lax function is defined in terms of superfields, it involves only bosonic momenta and the conserved charges are obtained from the bosonic residues of powers of the Lax function. Furthermore, in the dispersionless limit, we know that $\partial \rightarrow p$. However, the reduction of the fermionic covariant derivative in the dispersionless limit is not well understood. It is also already noted [20 $\left.\overline{2}_{1}\right]$ that a scaling of the fermionic covariant derivative is essential in order to preserve the supersymmetry in the dispersionless limit. In view of the above mentioned difficulties, our strategy, as a first step, is to look at supersymmetric systems which are described in terms of Lax operators that involve only bosonic $\partial$ operators.

For one of the three known families of integrable supersymmetric hierarchies with $N=2, W_{n}$ superalgebra as the second Hamiltonian structure, the Lax operators contain only bosonic operators of the forms [26]:

$$
L_{s}=\partial-\left[D \frac{1}{\partial^{s}+\sum_{i=1}^{s} J_{i} \partial^{s-i}} \bar{D}\left(\sum_{i=1}^{s} J_{i} \partial^{s-i}\right)\right] .
$$

Here, $s=0,1,2, \ldots$ and $J_{i}$ are bosonic $N=2$ superfields of dimensions $i$. Furthermore, the square brackets stand for the fact that the $N=2$ supersymmetric fermionic covariant 
derivatives $D$ and $\bar{D}$, defined to be

$$
D=\frac{\partial}{\partial \theta}-\frac{\bar{\theta}}{2} \partial, \quad \bar{D}=\frac{\partial}{\partial \bar{\theta}}-\frac{\theta}{2} \partial
$$

act only on the superfields inside the brackets.

Let us consider the conventional dispersionless limit in the simplest case of the $N=2$ supersymmetric KdV hierarchy with $s=1$. The Lax operator, in this case, has the form

$$
L=\partial-\left[D \frac{1}{\partial+J} \bar{D} J\right]
$$

The second flow, following from this Lax operator, reads

$$
J_{t_{2}}=\left([D, \bar{D}] J-J^{2}\right)_{x} .
$$

Under the rescaling $\partial_{t} \rightarrow \lambda \partial_{t}, \partial \rightarrow \lambda \partial,(D, \bar{D}) \rightarrow \lambda^{\frac{1}{2}}(D, \bar{D})$, this equation will reduce to $($ as $\lambda \rightarrow 0)$

$$
J_{t_{2}}=-\left(J^{2}\right)_{x}
$$

However, this equation, despite being $N=2$ supersymmetric, is not very interesting.

A different possibility consists in rescaling to rescale in a standard way all the fields together with the fermionic derivatives in the Lax operator $\left(\overline{6} \cdot \overline{3}_{1}\right)$. This leads to the following Lax function in the dispersionless limit:

$$
\mathcal{L}=p-\frac{\frac{1}{2} \mathcal{T}}{p+\mathcal{J}}-\frac{\frac{1}{2} \psi_{1} \psi_{2}}{(p+\mathcal{J})^{2}}
$$

where we introduced the component fields:

$$
\mathcal{J}=J\left|, \psi_{1}=(D+\bar{D}) J\right|, \psi_{2}=(D-\bar{D}) J|, \mathcal{T}=[D, \bar{D}] J|,
$$

and the restriction $\mid$ stands for keeping the $(\theta=\bar{\theta}=0)$ term. One can check, that the second flow equations

$$
\begin{aligned}
& \mathcal{J}_{t_{2}}=\left(\mathcal{T}-\mathcal{J}^{2}\right)_{x}, \quad \mathcal{T}_{t_{2}}=-2\left(\mathcal{J} \mathcal{T}-\psi_{1} \psi_{2}\right)_{x}, \\
& \left(\psi_{1}\right)_{t_{2}}=-2\left(\mathcal{J} \psi_{1}\right)_{x}, \quad\left(\psi_{2}\right)_{t_{2}}=-2\left(\mathcal{J} \psi_{2}\right)_{x}
\end{aligned}
$$

do not possess any supersymmetry at all. They break even the $N=1$ supersymmetry. The same is also true for higher flows.

Therefore, we propose the following alternative approach to take the dispersionless limit. The main idea is to rescale the fermionic components of the superfields $J_{i}$ differently from the conventional method as

$$
\begin{aligned}
& J_{i}\left|\rightarrow \alpha^{i} J_{i}\right|,(D+\bar{D}) J_{i}\left|\rightarrow \alpha^{i}(D+\bar{D}) J_{i}\right| \\
& (D-\bar{D}) J_{i}\left|\rightarrow \alpha^{i+1}(D-\bar{D}) J_{i}\right|,[D, \bar{D}] J_{i}\left|\rightarrow \alpha^{i+1}[D, \bar{D}] J_{i}\right|
\end{aligned}
$$

It is clear that this unconventional, alternative rescaling (6. $N=2$ supersymmetry. However, a subset of $N=1$ supersymmetry, generated by $(D+\bar{D})$, 
will survive and, in the dispersionless limit, we will have a Lax description for an $N=1$ supersymmetric system of equations.

Let us demonstrate in detail how all this works for the Lax operator (6.3. to our alternative procedure, the first step will consist of representing $\frac{1}{\partial+J}$ as

$$
\frac{1}{\partial+J} \equiv \partial^{-1}+A_{2} \partial^{-2}+A_{3} \partial^{-3}+A_{4} \partial^{-4}+\ldots
$$

where all the functions $A_{n}$ can be recursively calculated and the first few have the explicit forms

$$
A_{2}=-J, A_{3}=J^{2}+J_{x}, A_{4}=-J^{3}-3 J J_{x}-J_{x x}, \ldots .
$$

Thus, our Lax operator can also be written as

$$
L=\partial-\left[D\left(\partial^{-1} \bar{D} J+A_{2} \partial^{-2} \bar{D} J+A_{3} \partial^{-3} \bar{D} J+A_{4} \partial^{-4} \bar{D} J+\ldots\right)\right],
$$

and we should move the partial derivatives to the right in $(6.12 i)$.

The first non trivial term on the right hand side of (6.12i) generates an infinite series of terms when the derivative is moved to the right, namely,

$$
\partial^{-1}[D \bar{D} J] \equiv \frac{1}{2}\left(\mathcal{T}-\mathcal{J}_{x}\right) \partial^{-1}-\frac{1}{2}\left(\mathcal{T}-\mathcal{J}_{x}\right)_{x} \partial^{-2}+\frac{1}{2}\left(\mathcal{T}-\mathcal{J}_{x}\right)_{x x} \partial^{-3}+\ldots
$$

We may now replace $\partial \rightarrow p$ in the r.h.s. of (6.13i) and rescale

$$
p \rightarrow \alpha p, \mathcal{J} \rightarrow \alpha \mathcal{J}, \psi_{1} \rightarrow \alpha \psi_{1}, \psi_{2} \rightarrow \alpha^{2} \psi_{2}, \mathcal{T} \rightarrow \alpha^{2} \mathcal{T} .
$$

Then, it is easy to see that the only term among those in (6.13) that will contribute to $\lim _{\alpha \rightarrow \infty} \frac{1}{\alpha} L_{\alpha}$ is

$$
\frac{1}{2} \mathcal{T} p^{-1}
$$

The second term inside the square bracket in the right hand side of (6.12i) needs some more work:

$$
\begin{gathered}
\left(D A_{2}\right) \partial^{-2}(\bar{D} J)+A_{2} \partial^{-2}(D \bar{D} J) \equiv\left(D A_{2}\right)(\bar{D} J) \partial^{-2}-2\left(D A_{2}\right)(\bar{D} J)_{x} \partial^{-3}+ \\
A_{2}(D \bar{D} J) \partial^{-2}-2 A_{2}(D \bar{D} J)_{x} \partial^{-3}+\ldots,
\end{gathered}
$$

where the dots stand for terms with $\partial^{-4}$ and higher. In the scaling limit, only the following terms will survive

$$
\frac{1}{2} \psi_{1} \psi_{2} p^{-2}-\frac{1}{2} \psi_{2}\left(\psi_{2}\right)_{x} p^{-3}-\frac{1}{2} \mathcal{J} \mathcal{T} p^{-2} .
$$

Continuing in a similar manner, we find the Lax operator in the dispersionless limit to be

$$
\mathcal{L}=p-\frac{\frac{1}{2} \mathcal{T}}{p+\mathcal{J}}-\frac{\frac{1}{2} \psi_{1} \psi_{2}}{(p+\mathcal{J})^{2}}+\frac{\frac{1}{4} \psi_{2}\left(\psi_{2}\right)_{x}}{(p+\mathcal{J})^{3}}
$$

The second flow, following from the Lax equation, has the form

$$
\begin{aligned}
& \mathcal{J}_{t_{2}}=\left(\mathcal{T}-\mathcal{J}^{2}\right)_{x}, \quad \mathcal{T}_{t_{2}}=-2\left(\mathcal{J} \mathcal{T}-\psi_{1} \psi_{2}\right)_{x} \\
& \left(\psi_{1}\right)_{t_{2}}=\left(\left(\psi_{2}\right)_{x}-2 \mathcal{J} \psi_{1}\right)_{x}, \quad\left(\psi_{2}\right)_{t_{2}}=-2\left(\mathcal{J} \psi_{2}\right)_{x}
\end{aligned}
$$


These equations can also be easily rewritten in terms of $N=1$ superfields,

$$
j=\mathcal{J}+\theta \psi_{1}, \quad \psi=\psi_{2}-\theta \mathcal{T}
$$

as

$$
j_{t_{2}}=-\left(\mathcal{D} \psi+j^{2}\right)_{x}, \quad \psi_{t_{2}}=-2(j \psi)_{x},
$$

where

$$
\mathcal{D}=\frac{\partial}{\partial \theta}-\theta \partial, \quad \mathcal{D}^{2}=-\partial .
$$

Let us note here that the Lax operator $(6 . \overline{1} \overline{6})$ is not new and is gauge equivalent to the one which has been constructed earlier by brute force in [20 $2 \overline{2}]$. However, we see that it can be systematically obtained from the alternative dispersionless limit of the simplest of the Lax operators in the family $\left(\overline{6}_{1} \cdot \overline{1}_{1}\right)$.

\section{Supersymmetric Boussinesq hierarchy}

As a second example of our method, in this section, we will work out the dispersionless limit starting from the $N=2$ supersymmetric Boussinesq hierarchy with $\alpha=\frac{5}{2}$ [27], which is described by the Lax operator $\left(\overline{6}_{\overline{6}} \overline{1}_{1}\right)$ with $s=2[26]$. The Lax operator $\left(\overline{6} \cdot \overline{1}_{1}^{\prime}\right)$, in this case, has the explicit form

$$
L=\partial-\left[D \frac{1}{\partial^{2}+J_{1} \partial+J_{2}} \bar{D}\left(J_{1} \partial+J_{2}\right)\right]
$$

Following our procedure, we will first rewrite

$$
\frac{1}{\partial^{2}+J_{1} \partial+J_{2}}=\partial^{-2}+A_{1} \partial^{-3}+A_{2} \partial^{-4}+\ldots,
$$

where all the $A_{n}$ 's can be easily calculated,

$$
A_{1}=-J_{1}, A_{2}=-J_{2}+2\left(J_{1}\right)_{x}+J_{1}^{2}, A_{3}=\left(2 J_{2}-3\left(J_{1}\right)_{x}-\frac{5}{2} J_{1}^{2}\right)_{x}+2 J_{1} J_{2}-J_{1}^{3}, \cdots
$$

With this, the first few terms in the square bracket in $\left(\begin{array}{l}1 \\ 1\end{array}\right]$ ) have the form

$$
\begin{aligned}
{\left[D \frac{1}{\partial^{2}+J_{1} \partial+J_{2}} \bar{D}\left(J_{1} \partial+J_{2}\right)\right]=} & {\left[D \partial^{-2} \bar{D}\left(J_{1} \partial+J_{2}\right)\right]+\left[D A_{1} \partial^{-3} \bar{D}\left(J_{1} \partial+J_{2}\right)\right]+(7.4) } \\
& {\left[D A_{2} \partial^{-4} \bar{D}\left(J_{1} \partial+J_{2}\right)\right]+\left[D A_{3} \partial^{-5} \bar{D}\left(J_{1} \partial+J_{2}\right)\right]+\ldots }
\end{aligned}
$$

Let us next introduce the components

$$
\begin{aligned}
& \mathcal{J}_{1}=J_{1}\left|, \psi_{1}=(D+\bar{D}) J_{1}\right|, \psi_{2}=(D-\bar{D}) J_{1}\left|, \mathcal{T}_{1}=[D, \bar{D}] J_{1}\right|, \\
& \mathcal{T}_{2}=J_{2}\left|, \xi_{1}=(D+\bar{D}) J_{2}\right|, \xi_{2}=(D-\bar{D}) J_{2}\left|, \mathcal{W}=[D, \bar{D}] J_{2}\right|
\end{aligned}
$$

which have the scaling behaviors:

$$
\left(\mathcal{J}_{1}, \psi_{1}\right) \rightarrow \alpha\left(\mathcal{J}_{1}, \psi_{1}\right),\left(\psi_{2}, \mathcal{T}_{1}, \mathcal{T}_{2}, \xi_{1}\right) \rightarrow \alpha^{2}\left(\psi_{2}, \mathcal{T}_{1}, \mathcal{T}_{2}, \xi_{1}\right),\left(\xi_{2}, \mathcal{W}\right) \rightarrow \alpha^{3}\left(\xi_{2}, \mathcal{W}\right)
$$

We are now ready to find a Lax function in the dispersionless limit. 
We can now have the fermionic derivatives act on the fields in (i 7.4$)$, move the partial derivatives to the right and replace $\partial \rightarrow p$. After this, it is easy to see that there will be three types of terms that may survive in the limit ( $\left.{ }^{2} .3 \overline{3}\right)$ :

$$
\begin{aligned}
\mathcal{L} & \equiv p-\mathcal{A}-\mathcal{B}-\mathcal{C} \\
\mathcal{A} & \equiv \frac{1}{2}\left(\mathcal{T}_{1} p+\mathcal{W}\right)\left(p^{-2}+A_{1} p^{-3}+A_{2} p^{-4}+\ldots\right) \\
\mathcal{B} & \equiv\left(\left(D A_{1}\right) p^{-3}+\left(D A_{2}\right) p^{-4}+\ldots\right)\left(\left(\bar{D} J_{1}\right) p+\left(\bar{D} J_{2}\right)\right) \\
\mathcal{C} & \equiv\left(-3\left(D A_{1}\right) p^{-4}-4\left(D A_{2}\right) p^{-5}+\ldots\right)\left(\left(\bar{D} J_{1}\right) p+\left(\bar{D} J_{2}\right)\right)_{x} .
\end{aligned}
$$

Note that the expressions in the parenthesis for $\mathcal{A}, \mathcal{B}, \mathcal{C}$ contain terms with and without derivatives (see eq. $\left.\left(\overline{7} \cdot \overline{1}_{i}\right)\right)$. For terms of the types $\mathcal{A}$ and $\mathcal{C}$ there is no problem, since in the dispersionless limit (scaling limit), only terms without derivatives in $A_{n}\left(\underline{\overline{7}}, \bar{B}_{i}\right)$ contribute. In this case, we have:

$$
\mathcal{A}=\frac{\frac{1}{2}\left(\mathcal{T}_{1} p+\mathcal{W}\right)}{p^{2}+\mathcal{J}_{1} p+\mathcal{T}_{2}}, \quad \mathcal{C}=-\left[D \frac{2 p+J_{1}}{\left(p^{2}+J_{1} p+J_{2}\right)^{2}}\right]\left(\left(\bar{D} J_{1}\right) p+\left(\bar{D} J_{2}\right)\right)_{x} .
$$

However, for terms of the type $\mathcal{B}$, the scaling require us to keep also the terms linear in the first derivatives in all the $A_{n}$ 's. This leads to

$$
\mathcal{B}=\left[D\left(\frac{1}{p^{2}+J_{1} p+J_{2}}+\frac{\left(2 p+J_{1}\right)\left(J_{1} p+J_{2}\right)_{x}}{\left(p^{2}+J_{1} p+J_{2}\right)^{3}}\right)\right]\left(\left(\bar{D} J_{1}\right) p+\left(\bar{D} J_{2}\right)\right) .
$$

In the dispersionless limit the Lax function now becomes

$$
\begin{aligned}
\mathcal{L}= & p-\frac{\frac{1}{2}\left(\mathcal{T}_{1} p+\mathcal{W}\right)}{p^{2}+\mathcal{J}_{1} p+\mathcal{T}_{2}}-\frac{\frac{1}{4} \psi_{2}\left(\psi_{2} p+\xi_{2}\right)_{x}}{\left(p^{2}+\mathcal{J}_{1} p+\mathcal{T}_{2}\right)^{2}}-\frac{\frac{1}{2}\left(\psi_{1} p+\xi_{1}\right)\left(\psi_{2} p+\xi_{2}\right)}{\left(p^{2}+\mathcal{J}_{1} p+\mathcal{T}_{2}\right)^{2}}+ \\
& \frac{\frac{1}{4}\left(2 p+\mathcal{J}_{1}\right)\left(\psi_{2} p+\xi_{2}\right)\left(\psi_{2} p+\xi_{2}\right)_{x}}{\left(p^{2}+\mathcal{J}_{1} p+\mathcal{T}_{2}\right)^{3}}+\frac{\frac{1}{4}\left(\mathcal{J}_{1} p+\mathcal{T}_{2}\right)_{x} \psi_{2} \xi_{2}}{\left(p^{2}+\mathcal{J}_{1} p+\mathcal{T}_{2}\right)^{3}}
\end{aligned}
$$

It is now easy to check that the Lax equation ( 5.200 leads to the dispersionless supersymmetric Boussinesq hierarchy. Explicitly, the second flow of this hierarchy is given by

$$
\begin{aligned}
& \left(\mathcal{J}_{1}\right)_{t_{2}}=\left(2 \mathcal{T}_{1}+2 \mathcal{T}_{2}-\mathcal{J}_{1}^{2}\right)_{x} \\
& \left(\mathcal{T}_{1}\right)_{t_{2}}=2\left(\mathcal{W}-\mathcal{J}_{1} \mathcal{T}_{1}+\psi_{1} \psi_{2}\right)_{x},\left(\mathcal{T}_{2}\right)_{t_{2}}=-2\left(\mathcal{J}_{1}\right)_{x} \mathcal{T}_{2}+\mathcal{J}_{1}\left(\mathcal{T}_{1}\right)_{x} \\
& (\mathcal{W})_{t_{2}}=-2\left(\mathcal{J}_{1}\right)_{x} \mathcal{W}-2\left(\mathcal{T}_{1}\right)_{x} \mathcal{T}_{2}+\mathcal{T}_{1}\left(\mathcal{T}_{1}\right)_{x}+\psi_{2}\left(\psi_{2}\right)_{x x}+2\left(\xi_{1}\left(\psi_{2}\right)_{x}-\xi_{2}\left(\psi_{1}\right)_{x}\right) \\
& \left(\psi_{1}\right)_{t_{2}}=2\left(\xi_{1}+\left(\psi_{2}\right)_{x}-\mathcal{J}_{1} \psi_{1}\right)_{x},\left(\psi_{2}\right)_{t_{2}}=2\left(\xi_{2}-\mathcal{J}_{1} \psi_{2}\right)_{x} \\
& \left(\xi_{1}\right)_{t_{2}}=-2\left(\mathcal{J}_{1}\right)_{x} \xi_{1}-2 \mathcal{T}_{2}\left(\psi_{1}\right)_{x}+\left(\mathcal{T}_{1}\right)_{x} \psi_{1}+\mathcal{J}_{1}\left(\psi_{2}\right)_{x x} \\
& \left(\xi_{2}\right)_{t_{2}}=-2\left(\mathcal{J}_{1}\right) \xi_{2}-2 \mathcal{T}_{2}\left(\psi_{2}\right)_{x}+\left(\mathcal{T}_{1}\right)_{x} \psi_{2}
\end{aligned}
$$

This system of equations can also be rewritten in terms of $N=1$ superfields

$$
j_{1}=\mathcal{J}_{1}+\theta \psi_{1}, \eta_{1}=\psi_{2}-\theta \mathcal{T}_{1}, j_{2}=\mathcal{T}_{2}+\theta \xi_{1}, \eta_{2}=\xi_{2}-\theta \mathcal{W}
$$

as

$$
\begin{aligned}
& \left(j_{1}\right)_{t_{2}}=\left(-2 \mathcal{D} \eta_{1}+2 j_{2}-j_{1}^{2}\right)_{x} \quad\left(\eta_{1}\right)_{t_{2}}=2\left(\eta_{2}-j_{1} \eta_{1}\right)_{x} \\
& \left(j_{2}\right)_{t_{2}}=-2\left(j_{1}\right)_{x} j_{2}-j_{1}\left(\mathcal{D} \eta_{1}\right)_{x},\left(\eta_{2}\right)_{t_{2}}=-2\left(j_{1}\right)_{x} \eta_{2}-2 j_{2}\left(\eta_{1}\right)_{x}-\left(\mathcal{D} \eta_{1}\right)_{x} \eta_{1} .
\end{aligned}
$$

Thus, we explicitly demonstrate that our system possesses an $N=1$ supersymmetry. 


\section{Acknowledgement}

The author would like to thank the organizers for the kind invitation and financial support during the conference.

\section{References}

[1] Yu. I. Manin, J. Sov. Math. 11 (1979) 1.

[2] B. A. Dubrovin, S. P. Novikov, Russian Math. Surveys 44 (1989) 35.

[3] V. E. Zakharov, Funct. Anal. Appl. 14 (1980) 89.

[4] J. Cavalcante, H. P. McKean, Physica D4 (1982) 253.

[5] P. J. Olver, Y. Nutku, J. Math. Phys. 29 (1988) 1610.

[6] G. B. Whitham, Linear and Nonlinear Waves, (Wiley, New York, 1974).

[7] M. Born, L. Infeld, Proc. R. Soc. London, A144 (1934) 425.

[8] B. M. Barbashov, N. A. Chernikov, Sov. Phys. JETP 24 (1966) 93; M. Arik, F. Neyzi, Y. Nutku, P. J. Olver, J. Verosky, J. Math. Phys. 30 (1988) 1338.

[9] D. Lebedeev, Yu. I. Manin, Phys. Lett. A74 (1979) 154; V. E. Zakharov, Physica D3 (1981) 193; Y. Kodama, J. Gibbons, Phys. Lett. A135 (1989) 167; I. M. Krichever, Comm. Math. Phys. 143 (1991) 415; K. Takasaki, T. Takabe, Rev. Math. Phys. 7 (1995) 743; A. M. Bloch, H. Flaschka, T. S. Ratiu, Comm. Fields Inst. 7 (1995) 57.

[10] E. Witten, Nucl. Phys. B340 (1990) 281; P. Dijkraaf, E. Verlinde, H. Verlinde, Nucl. Phys. B352 (1991) 59. I. Krichever, Comm. Math. Phys. 143 (1992) 627; B. Dubrovin, Nucl. Phys. B379 (1993) 627.

[11] C. Laberge and P. Mathieu, Phys. Lett. B215 (1988) 718.

[12] P. Mathieu, Phys. Lett. B203 (1988) 287;

P. Labelle and P. Mathieu, J. Math. Phys. 32 (1991) 923;

Z. Popowicz, Phys. Lett. A174 (1993) 411;

J. C. Brunelli and A. Das, Int. J. Mod. Phys. 10 (1995) 4563;

S. Krivonos and A. Sorin, Phys. Lett. B357 (1995) 94;

Z. Popowicz, J. Phys. A29 (1996) 4987;

L. Bonora, S. Krivonos and A. Sorin, Nucl. Phys. B477 (1996) 835;

E. Ivanov and S. Krivonos, Phys. Lett. A231 (1997) 75;

F. Delduc and L. Gallot, Comm. Math. Phys. 190 (1997) 395;

L. Bonora, S. Krivonos and A. Sorin, Lett. Math. Phys. 45 (1998) 63;

S. Krivonos, A. Pashnev and Z. Popowicz, Mod. Phys. Lett. A18 (1998) 1435.

[13] S. Krivonos and A. Sorin, Phys. Lett. A251 (1999) 109.

[14] E. Ivanov and S. Krivonos, Phys. Lett. B291 (1992) 63; B301 (1993) 454(E);

S. Bellucci, E. Ivanov, S. Krivonos and A. Pichugin, Phys. Lett. B312 (1993) 463.

[15] R. Jackiw, (A Particle Theorist's) Lectures on (Supersymmetric, Non-Abelian) Fluid Mechanics (and d-Branes), MIT-CTP 3000; T. S. Nyawelo, J. W. van Holten, S. Groot Nibbelink, hep-th/0104104.

[16] Y. Bergner, R. Jackiw, physics/0103092. 
[17] A. Das, Z. Popowicz, Phys. Lett. A296 (2002) 15.

[18] A. Das, S.Krivonos, Z.Popowicz Phys. Lett. A302 (2002) 87.

[19] J. C. Brunelli, A. Das, Phys. Lett. A235 (1997) 597.

[20] Y. Nutku, M. V. Pavlov, J. Math. Phys. 43 (2002) 1441.

[21] B. A. Kupershmidt and Yu. I. Manin, Func. Anal. Appl. 11 (1978) 188; ibid 12 (1978) 20;

V. Zakharov, Func. Anal. Appl. 14 (1980) 15;

B. A. Dubrovin and S. P. Novikov, Sov. Math. Dokl. 27 (1983) 665;

D. B. Fairlie and I. A. Strachan, Inverse Probl. 12 (1998) 885;

S. Aoyama and Y. Kodama, Comm.Math.Phys. 182 (1996) 185.

[22] B.A. Kupershmidt, Comm.Math.Phys. 99 (1985) 51;

H.Aratyn, L.A.Ferreira, J.F.Gomes.R.T.Medeirosa, A.H.,Zimerman Mod.Phys.Lett A8 (1993) 3079

[23] L.Bonora, C.S.Xiong Phys¿Lett B285 (1992) 191, Phys.Lett b317 (1993) 329

[24] J. Barcelos-Neto, A. Constandache and A. Das, Phys. Lett. A268 (2000) 342;

A. Das and Z. Popowicz, J. Phys. A34 (2001) 6105;

A. Das and Z. Popowicz, Phys. Lett. A. 296 (2002) 15.

[25] A. Das and Z. Popowicz, Phys. Lett. A272 (2000) 65.

[26] S. Krivonos A. Sorin Phys.Lett A251 (1999) 109.

[27] E.Ivanov, S.Krivonos Phys.Lett B291 (1992) 63, B301 (1993) 454.

[28] Z.Popowicz Phys.Lett B459 (1999) 150. 